\title{
Natural frequncies of coupled blade-bending and shaft-torsional vibrations
}

\author{
B.O. Al-Bedoor* \\ Mechanical Engineering Department, Faculty of Engineering and Technology, University of Jordan, P.O. Box \\ 13568, Amman 11942, Jordan \\ E-mail: albedoor@ju.edu.jo
}

Received 11 June 2005

Revised 19 January 2006

\begin{abstract}
In this study, the coupled shaft-torsional and blade-bending natural frequencies are investigated using a reduced order mathematical model. The system-coupled model is developed using the Lagrangian approach in conjunction with the assumed modes method to discretize the blade bending deflection. The model accounts for the blade stagger (setting) angle, the system rotating speed and its induced stiffening effect. The coupled equations of motion are linearized based on the small deformation theory for the blade bending and shaft torsional deformation to enable calculation of the system natural frequencies for various combinations of system parameters. The obtained coupled eignvalue system is ready for use as a reference for comparison for larger size finite element simulations and for the use as a fast check on natural frequencies for the coupled blade bending and shaft torsional vibrations in the design and diagnostics processes. Some results on the predicted natural frequencies are graphically presented and discussed pertinent to the coupling controlling factors and their effects. In addition, the predicted coupled natural frequencies are validated using the Finite Element Commercial Package (Pro-Mechanica) where good agreements are found.
\end{abstract}

\section{Introduction}

The mathematical prediction of rotor system natural frequencies and mode shapes has contributed successfully in the analysis processes for design, retrofitting and diagnostics purposes. As major components that have direct interaction with the loading environment and in addition to the serious difficulties in measuring their vibrations, rotating blades have received special attention. The prediction of rotating blades natural frequencies has been, in most of the cases, done with isolation from the main rotor dynamics using mainly the finite element method, which is known as a costly numerical method and may produce huge amount of results that are some times difficult to interpret. Recently, the dynamic interaction between blade bending and shaft torsional vibrations has been recognized, theoretically and experimentally. A conclusion was reached that using a reduced order model will allow more understanding of the coupled blade bending and shaft torsional vibrations. Utilizing this dynamic interaction for predicting the rotating blade-shaft system natural frequencies as a coupled system is needed as a tool for evaluating the effect of this coupling on the system natural frequencies. This prediction should enable the investigation of effects of different shaft-disk-blade system parameters including blade stagger angle (setting angle) and will yield more realistic predictions of the coupled system natural frequencies.

The effect of rotation on the natural frequencies and mode shapes of a rotating structure has been investigated for many years resulting in numerous studies such as [1-3]. The main result of these studies was the strengthening effect that rotation has in the form of raising the structural natural frequencies due to the energy stored as a result of centrifugal force and beam shortening. Crawley and Mokadam [4] studied the inertial and elastic coupling of

\footnotetext{
* On Leave from King Fahd University of Petroleum \& Minerals, P. O. Box 841, Dhahran 31261, Saudi Arabia.
} 
non-rotating bladed disks. They used the Ritz analysis that enabled the identification of non-dimensional frequency and mass ratios that govern the bladed-disk-shaft coupling. Their results showed strong dependence of the system coupling on the blade stagger angle, blade-disk frequency ratio and mass ratios. Crawley et al. [5] studied the coupled bladed disk shaft whirl of a rotating cantilever turbofan. The model was built on the previous work of reference [4], but applied to a rotating system. They reported the phenomena of forward and backward whirl of the system as a function of speed of rotation. In the work of [4,5], no special attention was given to the coupling between the shaft torsional and blade bending vibrations. Okabe et al. [6] reported a study that emphasized the need for modeling the coupled blade bending and shaft torsional vibrations in turbo-machinery. They developed an equivalent model that coupled shaft-torsional and blade-tangential vibrations. The model adopted the modal synthesis procedure, wherein the blade was modeled as a simple mass-spring subsystem and the shaft as another discrete subsystem. The two subsystems were coupled and the natural frequencies were calculated. The model predictions were compared to measurement results for an actual turbo-machine and close agreement was found. In a step forward, where the blades were modeled using the assumed modes method, Huang and Ho [7] reported a study on the coupled shaft-torsional and blade bending vibrations of a bladed-disk assembly. They used the weighted residual method and the receptances at the connection between the disk and the blade-root to enforce the coupling. Due to the employed receptances technique, they identified some drawbacks in the numerical root finding algorithm for their eignvalue problem for certain combination of parameters. To reach a more realistic coupling between the shaft-torsional and blade-bending deformations, Al-Bedoor [8] developed a model utilizing the multibody dynamic approach. The model employed the finite element method to approximate the blade vibrations that identified the nonlinear dynamic interaction between the shaft torsional and blade bending vibrations. In a follow up study, a reduced order model was developed by Al-Bedoor [9]. The model employed the assumed modes method and the simulations showed the interaction in the start-up and the steady state operations of the shaft-disk-blade system. Moreover, it was observed that the frequencies of vibration of blades, in regions where they are expected to vibrate freely, are different than what were predicted for the isolated disk-blade system. Al-Bedoor et al. [10] used the ANSYS finite element package in addition to experimental results to study extracting blade vibration signature from the shaft torsional vibration signals. Huge amount of results was obtained and the effect of the coupling between blade-bending shaft-torsional vibrations could not be easily extracted.

As a result of the change in vibration frequencies of the rotating shaft-disk-blade system observed in references [9, 10], the pronounced effect of the stagger angle on the shaft-blade coupling reported in reference [4] and the dimensionality problem in the finite element models, the present study was motivated. The objective is studying the coupled natural frequencies using a reduced order model that is limited to uniform blade bending and shaft torsional vibrations.

A mathematical model for the coupled shaft-torsional and blade-bending vibrations is developed. The system is assumed to be rotating at constant speed driven by a motor through a flexible coupling that allows torsional flexibility. The blade root is attached to the rigid disk with stagger (setting) angle to simulate the lead-lag (tangential) and the flapping (umbrella) vibration modes. The Euler's Bernoulli Beam theory, in conjunction with the Assumed Modes Method, is utilized in approximating the blade vibration. The equations of motion in the non-rotating frame (Newtonian) are obtained using Lagrangian mechanics. In addition, the blade stiffening due to centripetal force and blade shortening are accounted for. The obtained equations are linearized and treated as a generalized eignvalue problem from which the natural frequencies for various combinations of shaft-disk-blade parameters are calculated. The model was simulated using the Pro-Mechanica Finite Element Package to enable validating the reduced order model prediction.

\section{Model development}

The idealized shaft-disk-blade system is shown in the schematic of Fig. 1. The disk is assumed to be rigid with radius $R_{d}$ and mass $M_{d}$ and the flexible blades are attached radially to the disk with a stagger angle $\beta$ measured from the axis of rotation as shown in the top view of the system, Fig. 1(a). The beam is assumed to be inextensible and the Euler-Bernoulli beam theory is adopted. The beam is discretized using the Assumed Modes Method, AMM, and the mode shapes of a cantilever beam are used. The model adopts the small deformation theory for both blade-bending 


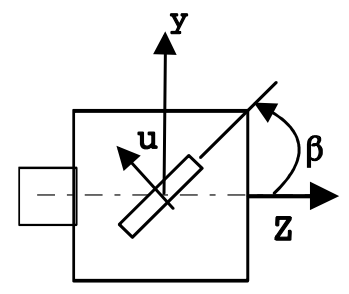

(a)
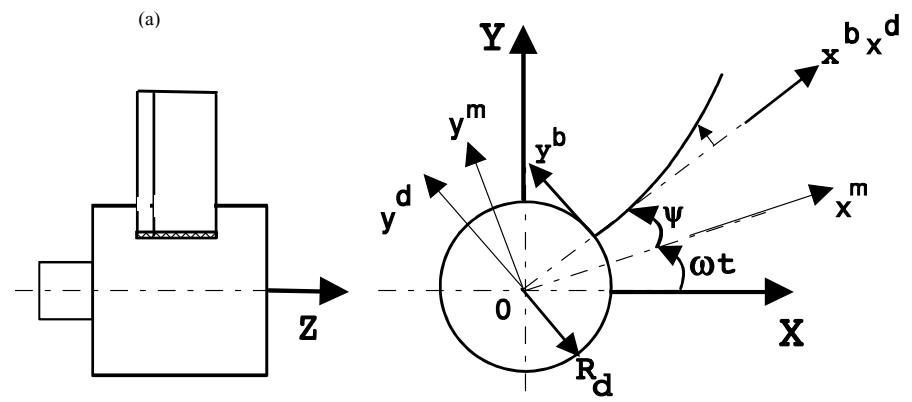

(b)

(c)

Fig. 1. Schematic of the disk-Blade system and the coordinate system.

and shaft-torsional deformations. The coordinate systems used in developing the model are shown in Fig. 1(c). Wherein, $X Y$ is the inertial reference frame, $x^{m} y^{m}$ is a body coordinate system of the motor shaft which is rotating at constant angular velocity $\omega, x^{d} y^{d}$ is a body coordinate system of the disk and $x^{b} y^{b}$ is the blade coordinate system that is attached to the root of the blade such that $x^{b}$ is always directed along the undeformed blade centerline. The body-coordinate system $x^{m} y^{m}$ and $x^{d} y^{d}$ have their origins at the origin of the inertial reference frame 0 while the blade coordinate system $x^{b} y^{b}$ has its origin at the disk circumference. Finally, $x y$ is the coordinate system that contains the blade transverse deflection $u(x, t)$ in the $y$ direction which in turn is related to the blade coordinate system $x^{b} y^{b}$ through the stagger (setting) angle $\beta$, as shown in Fig. 1(a).

\subsection{Kinetic energy expressions}

The kinetic energy of the shaft-disk-blade unit is constituted of the motor kinetic energy $U_{m}$, the disk kinetic energy $U_{d}$ and the blade kinetic energy $U_{b}$. The shaft inertia is lumped into the disk and motor inertia. The motor rotor and the disk are modeled as rigid inertia with mass moment of inertia $J_{m}$ and $J_{d}$ about the axis of rotation ( $Z$ ), respectively. As the system is rotating at a constant angular velocity $\omega$, the motor and disk kinetic energy expressions can be written, respectively, as

$$
\begin{aligned}
U_{m} & =\frac{1}{2} J_{m} \omega^{2} \\
U_{d} & =\frac{1}{2} J_{d}(\omega+\dot{\psi})^{2}
\end{aligned}
$$

where $\psi$ is the shaft torsional deformation angle measured with respect to the motor coordinate system as shown in Fig. 1(c).

To develop the kinetic energy expression of the blade, the deformed configuration of the shaft-disk-blade system, shown in Fig. 1(a) and (c), is used. The global position vector of a material point $P$, on the blade, can written as

$$
R_{P}=[A(\omega t)][A(\psi)] r_{P}^{d}
$$

where $r_{P}^{d}$ is the position vector of point $P$ in the disk coordinate system $x^{d} y^{d},[A(\omega t)]$ is the rotational transformation matrix from the motor coordinate system $x^{m} y^{m}$ to the inertial reference frame, $X Y$, and $[A(\psi)]$ is the rotational transformation matrix from the disk coordinate system, $x^{d} y^{d}$, to the motor coordinate system $x^{m} y^{m}$ which represents the angle of torsional deformation at the disk position. The position vector of the material point $P$ in the $x^{d} y^{d}$ 


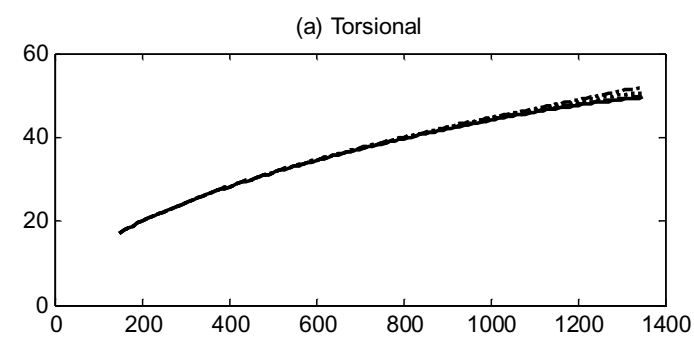

(c) Blade Second Bending Mode

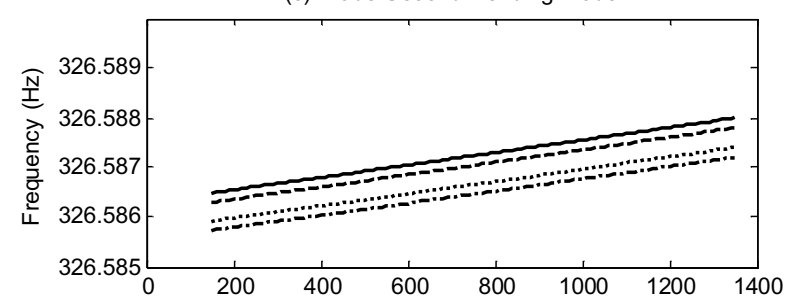

(e) Blade Fourth Bending Mode

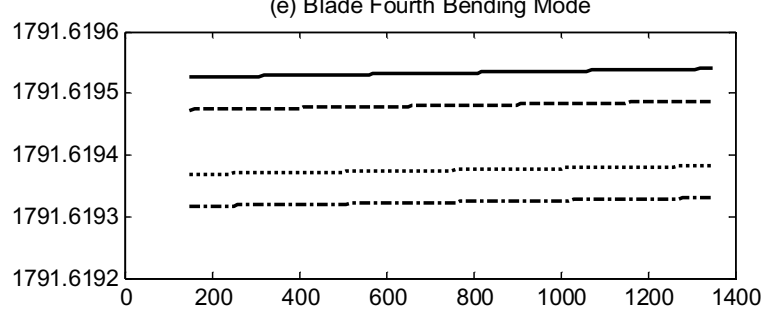

(b) Blade First Beding Mode

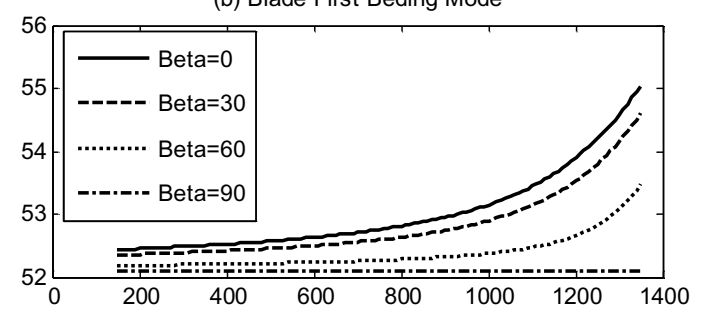

(d) Blade Third Bending Mode

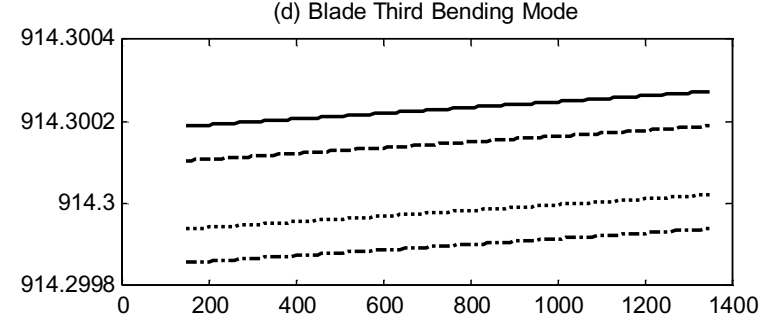

(f) Blade Fifth Bending Mode

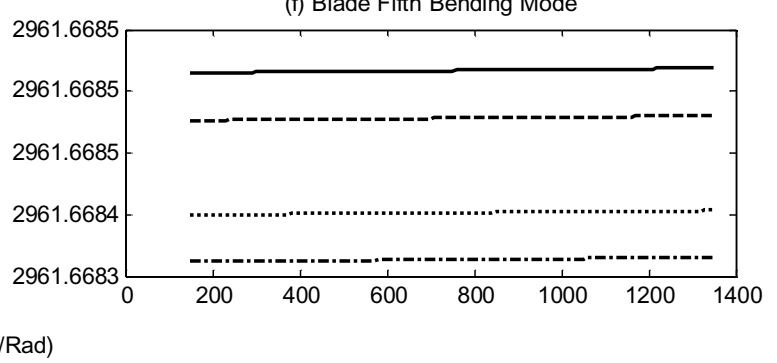

Fig. 2. Natural Frequencies as function of shaft torsional stiffness $k_{T}$ for setting angles: $\beta=0^{\circ}, 30^{\circ}, 60^{\circ}, 90^{\circ}$.

coordinate system can be written in the form

$$
r_{P}^{d}=\left(R_{d}+x\right) i+u(x, t) \cos \beta j+u(x, t) \sin \beta k
$$

where $R_{d}$ is the disk radius, at which the root of the blade is attached, $x$ and $u(x, t)$ are the axial position of the material point along the blade and the deflection as measured with respect to the coordinate system, $x y$. The rotational transformation matrices $[A(\omega t)]$ and $[A(\psi)]$ can be represented, respectively, as

$$
\begin{aligned}
{[A(\omega t)] } & =\left[\begin{array}{ccc}
\cos \omega t & -\sin \omega t & 0 \\
\sin \omega t & \cos \omega t & 0 \\
0 & 0 & 1
\end{array}\right] \\
{[A(\psi)] } & =\left[\begin{array}{ccc}
1 & -\psi & 0 \\
\psi & 1 & 0 \\
0 & 0 & 1
\end{array}\right]
\end{aligned}
$$

where $\omega$ represents the motor rigid body speed of rotation and $\psi$ represents the torsional deformation angle measured with respect to the motor coordinate system. The transformation matrix $[A(\psi)]$ is linearized based on the assumption of small torsional deformations.

The velocity vector of the material point in the inertial reference frame can be obtained by differentiating Eq. (2) that after manipulation can be represented as follows

$$
\dot{R}_{P}=[A(\omega t)][A(\psi)] \dot{r}_{P}^{d}+\left[\frac{d[A(\omega t)]}{d t}\right][A(\psi)] r_{P}^{d}+\dot{\psi}[A(\omega t)]\left[A_{\psi}(\psi)\right] r_{P}^{d}
$$

where $\left[A_{\psi}\right]$ represent the derivative $[d A / d \psi]$ of the matrix in Eq. (5). 
(a) Torsional

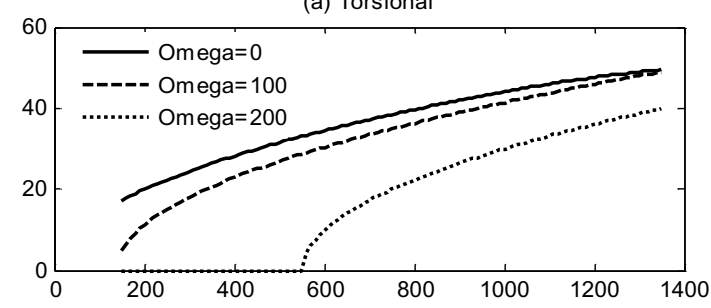

(c) Blade Second Bending Mode

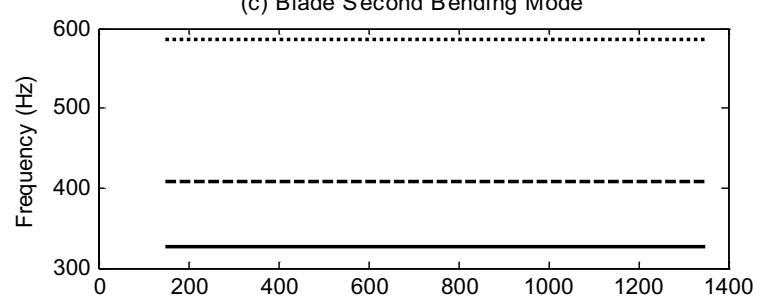

(e) Blade Fourth Bending Mode

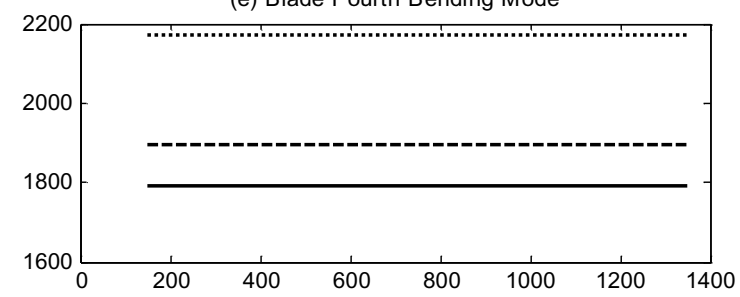

(b) Blade First Bending Mode

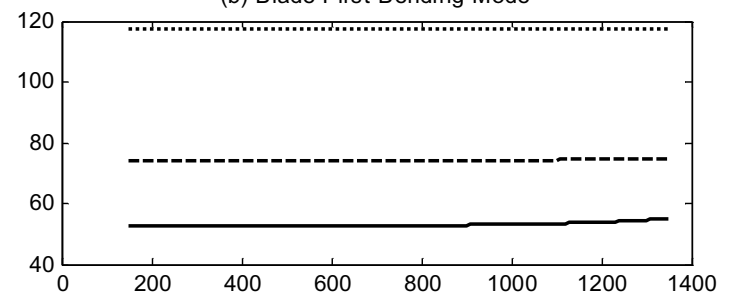

(d) Blade Thrid Bending Mode
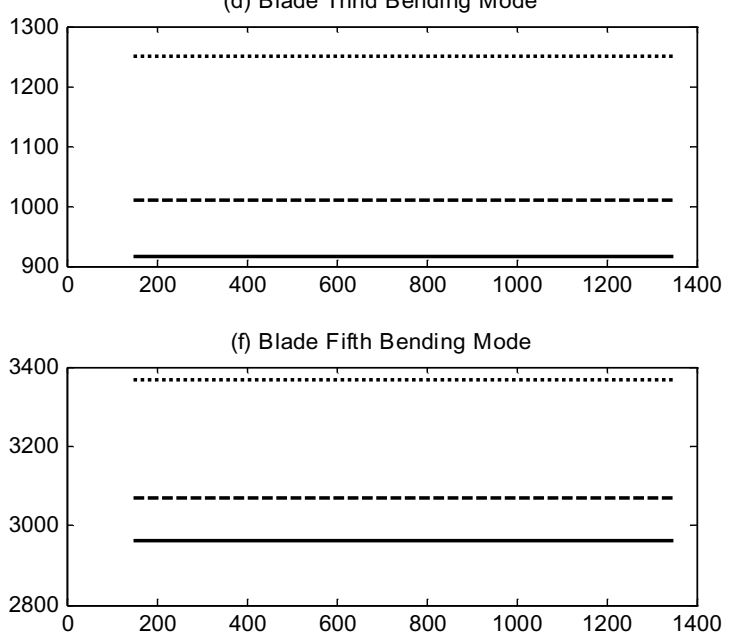

Kt (N.m/Rad)

Fig. 3. Natural Frequencies as function of shaft torsional stiffness $k_{T}$ for rotating speeds. $\omega=0,100,200 \mathrm{~Hz}$.

Upon differentiation, substitution and arrangement, Eq. (6), the velocity vector of the material point $P$ in the inertial reference frame can be represented in the form

$$
\dot{R}_{P}=\left\{\begin{array}{l}
-\Lambda_{1} \sin \omega t-\Lambda_{2} \cos \omega t \\
-\Lambda_{2} \sin \omega t+\Lambda_{1} \cos \omega t \\
-\dot{u} \sin \beta
\end{array}\right\}
$$

where

$$
\begin{aligned}
& \Lambda_{1}=\omega\left(R_{d}+x-\psi u \cos \beta\right)+\dot{\psi}\left(x+R_{d}\right)+\dot{u} \cos \beta \\
& \Lambda_{2}=R_{d} \psi \omega+\omega(x \psi+u \cos \beta)+\dot{\psi} u \cos \beta+\psi \dot{u} \cos \beta
\end{aligned}
$$

The kinetic energy of the blade can be found by using the velocity vector of Eq. (7) into the integral

$$
U_{b}=\frac{1}{2} \int_{0}^{L} \rho \dot{R}_{P}^{T} \dot{R}_{P}^{d} x
$$

where $\rho$ is the blade mass per unit length and $L$ is the blade length.

Now the total kinetic energy expression of the system can be written as follows

$$
U=U_{m}+U_{d}+U_{b}
$$

\subsection{Potential energy expressions}

The system potential energy is constituted of the blade bending strain energy, $V_{b}$, the torsional strain energy, $V_{T}$ and the potential energy of the axial shortening due to transverse deformations and the motion generated inertial 
(a) Torsional

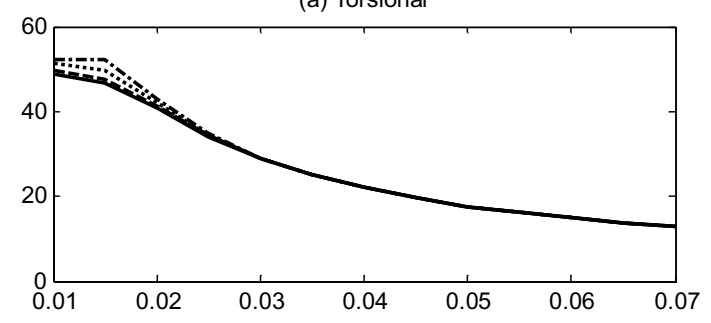

(c) Blade Second Bending Mode

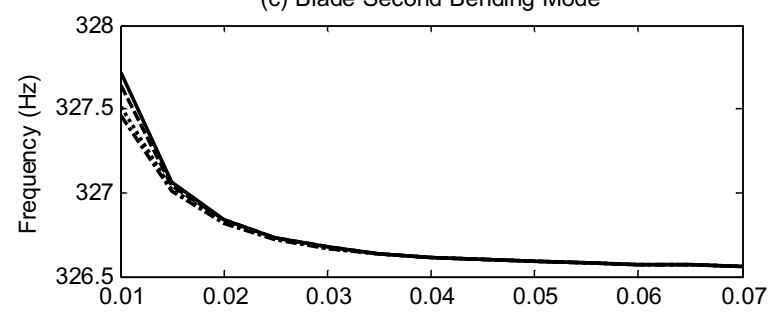

(e) Blade Fourth Bending Mode

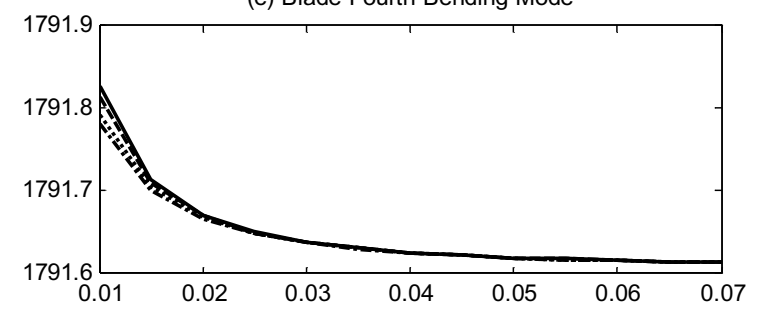

(b) Blade First Bending Mode

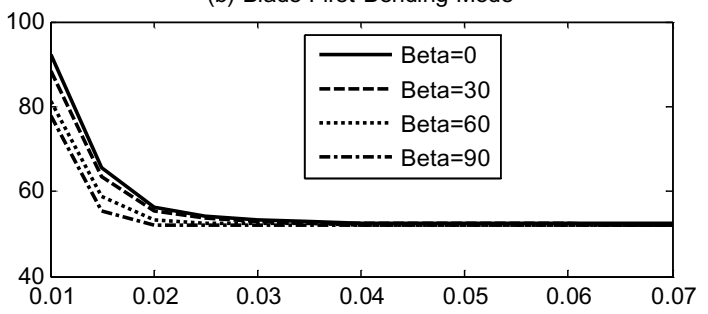

(d) Blade Third Bending Mode

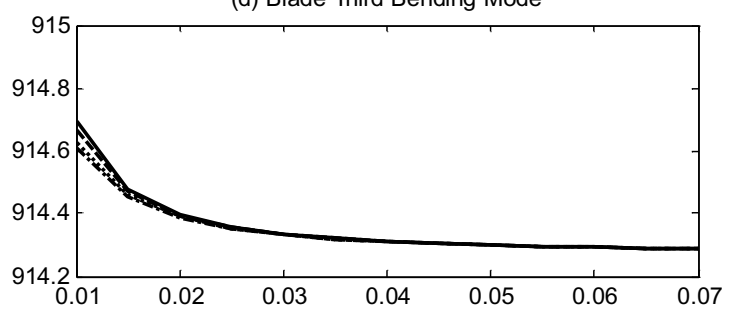

(f) Blade Fifth Bending Mode

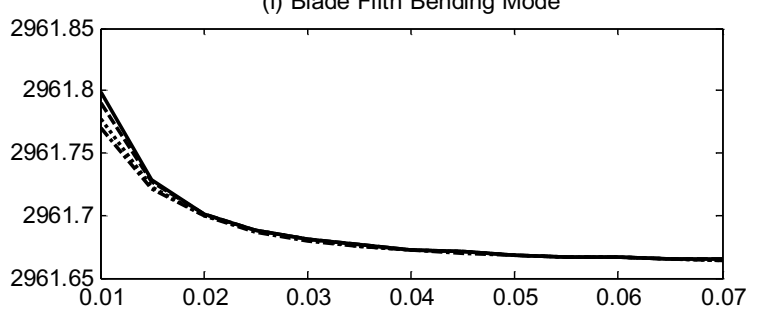

$\mathrm{RD}$ (meter)

Fig. 4. Natural Frequencies as function of shaft torsional stiffness $R_{D}$ for setting angles: $\beta=0^{\circ}, 30^{\circ}, 60^{\circ}, 90^{\circ}$.

forces, $V_{A}$. The elastic blade-bending strain energy for a blade with flexural rigidity $E I(x)$ is given by

$$
V_{b}=\frac{1}{2} \int_{0}^{L} E I(x) \frac{\partial^{2} u}{\partial x^{2}} d x
$$

, the torsional elastic potential energy stored in the flexible coupling is given by

$$
V_{T}=\frac{1}{2} k_{T} \psi^{2}
$$

The axial shortening due to transverse deformations in conjunction with the radial inertial forces contributes to the system elastic potential energy by the known axial shortening potential energy [9]. The axial shortening due to blade bending deformations can be approximated by

$$
d \delta \cong-\frac{1}{2}\left(\frac{\partial u}{\partial x}\right)^{2} d x
$$

The inertial force on the material point $P$ of the blade that results from the rotational motion of the blade can be expressed in the form

$$
F_{P}=\int_{x}^{L} \rho\left(R_{d}+x\right) \omega^{2} d x
$$

Now, the work that results from the axial shortening under the effect of the inertial forces can be called the axial shortening potential energy and can be written in the form 
(a) Torsional

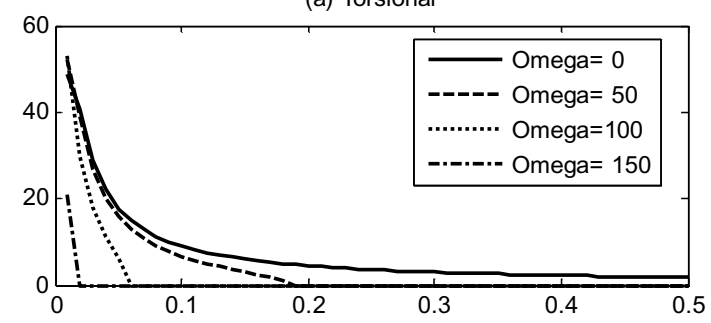

(c) Blade Second Bending Mode

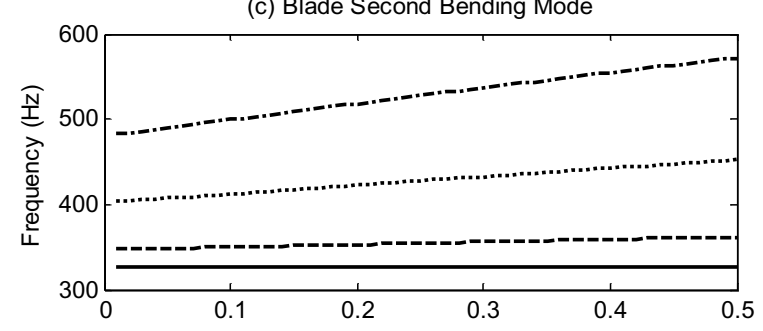

(e) Blade Fourth Bending Mode

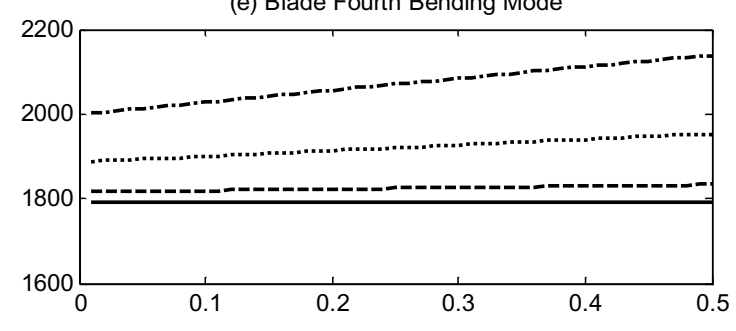

(b) Blade First Bending Mode

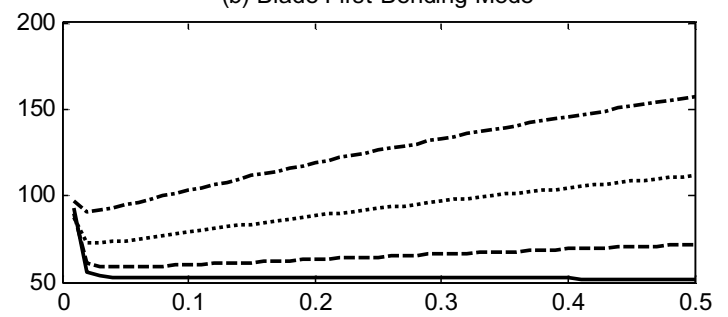

(d) Blade Third Bending Mode

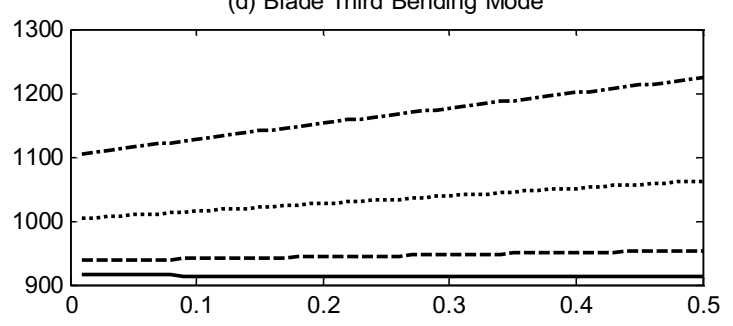

(f) Blade Fifth Bending Mode

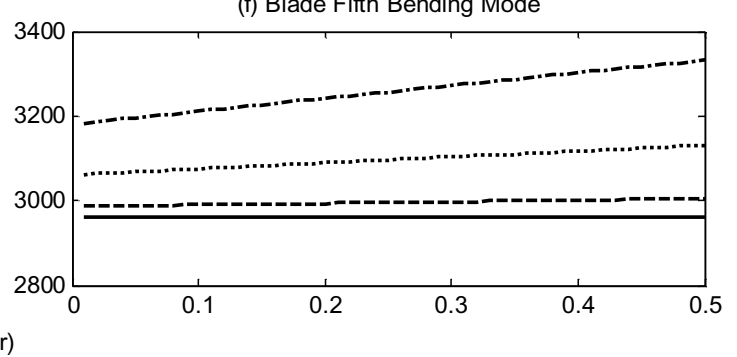

Fig. 5. Natural Frequencies as function of shaft torsional stiffness $R_{D}$ for rotating speeds: $\omega=0,50,100,150 \mathrm{~Hz}$.

$$
V_{A}=\int_{0}^{L} F_{P} d \delta
$$

Evaluating the integral of Eq. (14) and substituting for $F_{P}$ and $d \delta$ into Eq. (15), the axial shortening potential energy becomes

$$
V_{A}=\frac{1}{2} \omega^{2}\left\{\frac{1}{2} \int_{0}^{L} \rho\left(L^{2}-x^{2}\right)\left(\frac{\partial u}{\partial x}\right)^{2} d x+R_{d} \int_{0}^{L} \rho(L-x)\left(\frac{\partial u}{\partial x}\right)^{2} d x\right\}
$$

The system potential energy is

$$
V=V_{b}+V_{T}+V_{A}
$$

\subsection{The Assumed Modes Method (AMM)}

The assumed modes method is used in discretizing the blade elastic deformation, $u(x, t)$ shown in Fig. 1(c), relative to the blade coordinate system $x y$, as follows

$$
u(x, t)=\sum_{i=1}^{N} \phi_{i}(x) q_{i}(t)
$$

where $N$ is the number of modes, $q_{i}$ is the vector of modal coordinates, which is time dependent, and $\phi_{i}$ is the vector of the assumed modes. The mode shapes of a cantilever beam are used in this study. The mode shapes of a cantilever 
(a) Torsional

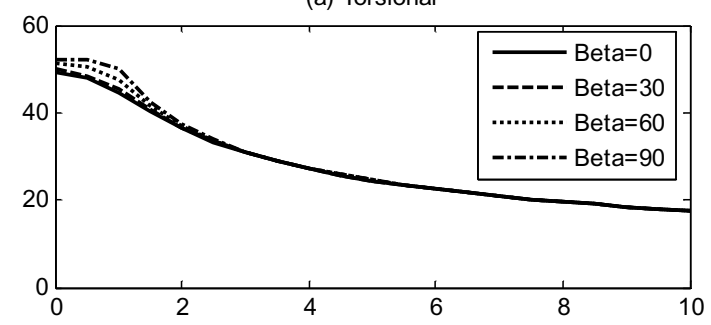

(c) Blade Second Bending Mode

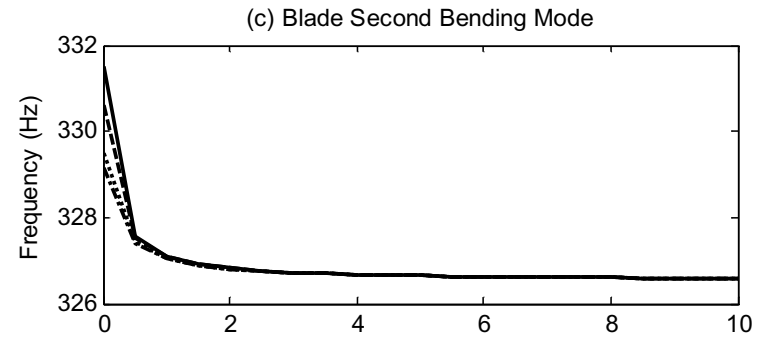

(e) Blade Fourth Bending Mode

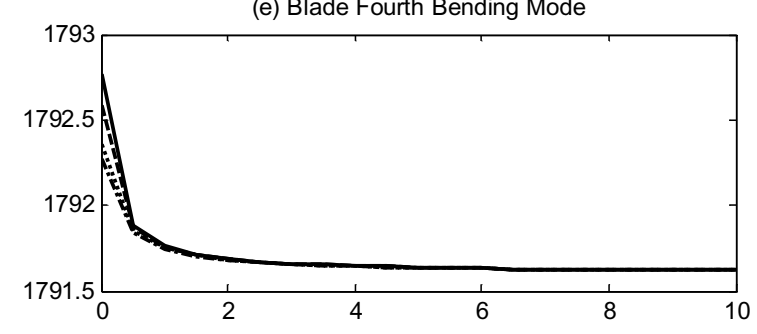

(b) Blade First Bending Mode

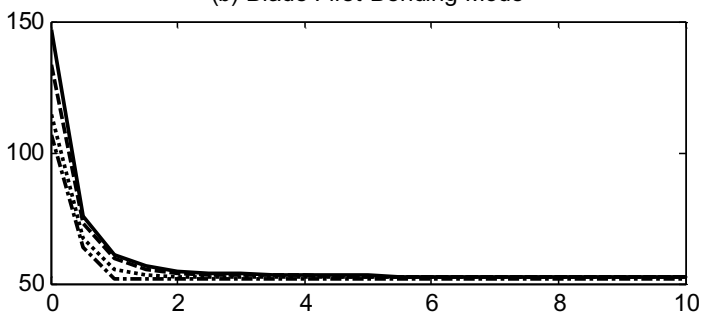

(d) Blade Third Bending Mode

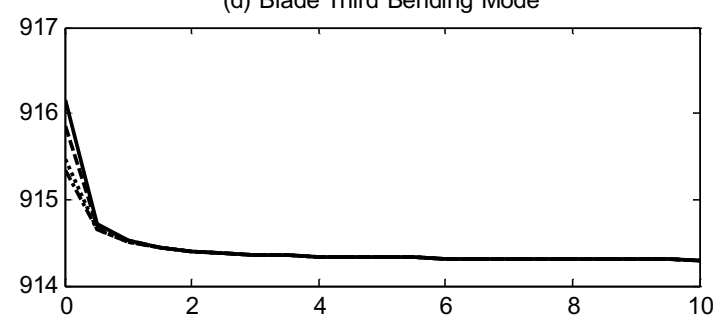

(f) Blade Fifth Bending Mode

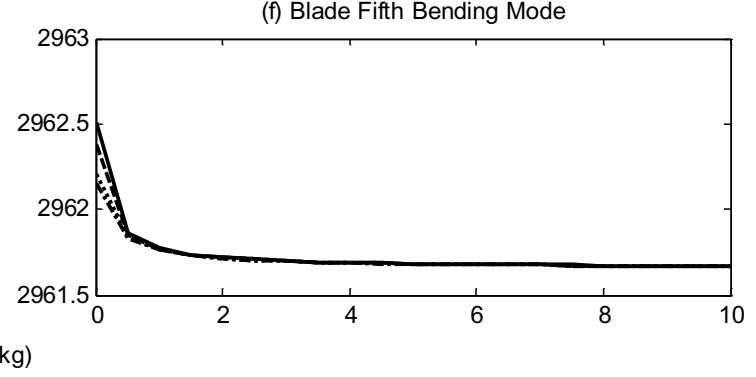

Fig. 6. Natural Frequencies as function of shaft torsional stiffness $M_{D}$ for setting angles: $\beta=0^{\circ}, 30^{\circ}, 60^{\circ}, 90^{\circ}$.

beam, which are normalized with respect to the mass per unit length of the blade, can be written in the from

$$
\phi_{i}(x)=\frac{1}{\sqrt{\rho L}}\left[\cosh \lambda_{i} x-\cos \lambda_{i} x-\alpha_{i}\left(\sinh \lambda_{i} x-\sin \lambda_{i} x\right)\right]
$$

where $\rho$ and $L$ are the blade mass per unit length and the blade length, respectively. The parameter $\lambda_{i}$ is found from the solution of the transcendental equation

$$
\cos \lambda_{i} L \cosh \lambda_{i} L+1=0
$$

and the parameter $\alpha_{i}=\frac{\sinh \lambda_{i} L-\sin \lambda_{i} L}{\cosh \lambda_{i} L+\cos \lambda_{i} L}$.

Upon substituting the AMM approximation for the blade deformations in the Lagrangian expression, a number of coefficient vectors and matrices are developed. The coefficient vectors and matrices [9], can be expressed as follows

$$
\begin{aligned}
& {[a]=\int_{0}^{L} \rho x[\phi(x)] d x} \\
& {[b]=\int_{0}^{L} \rho[\phi(x)] d x} \\
& {[I]=\int_{0}^{L} \rho[\phi(x)]^{T}[\phi(x)] d x}
\end{aligned}
$$


(a) Torsiona

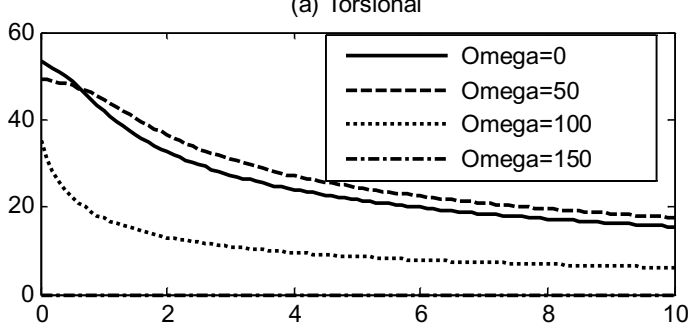

(c) Blade Second Bending Mode

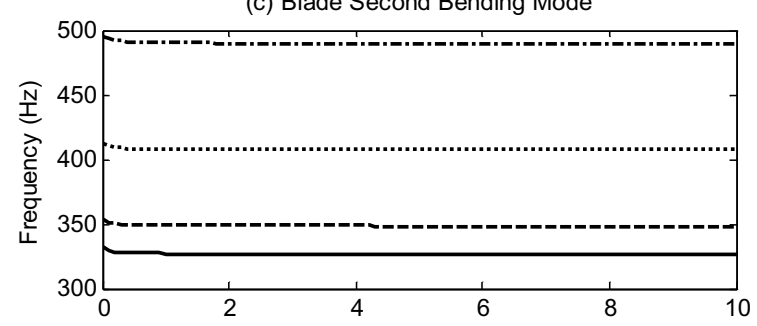

(e) Blade Fourth Bending Mode

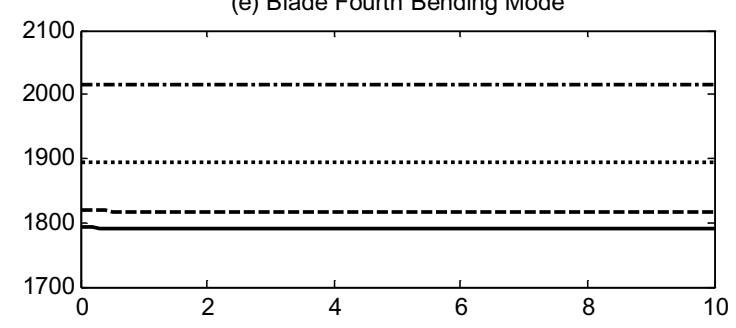

(b) Blade First Bending Mode

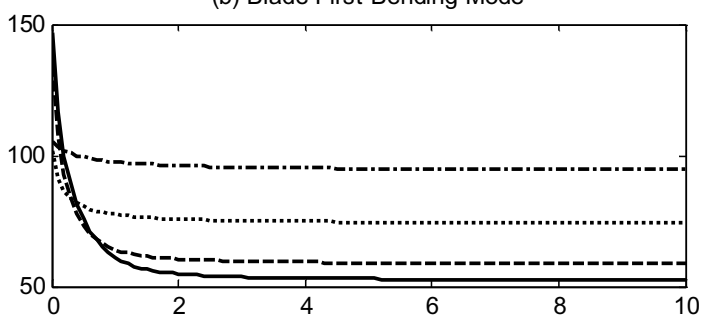

(d) Blade Third Bending Mode

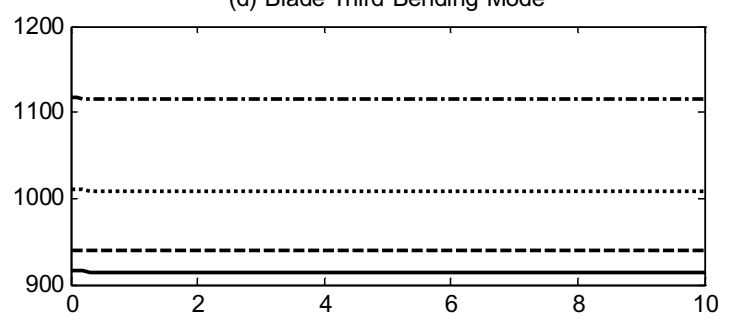

(f) Blade Fifth Bending Mode

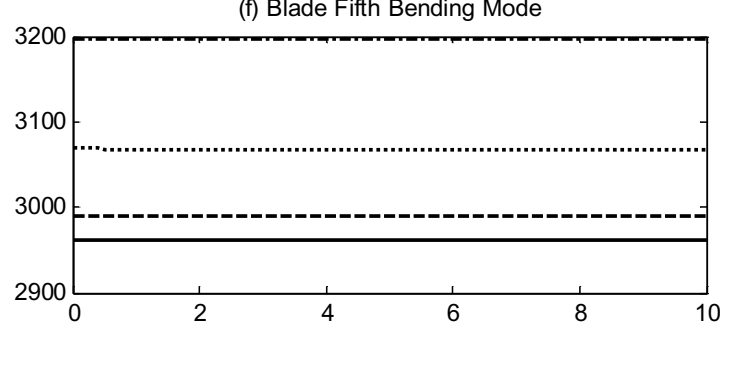

Fig. 7. Natural Frequencies as function of shaft torsional stiffness $M_{D}$ for rotating speeds: $\omega=0,50,100,150 \mathrm{~Hz}$.

$$
\begin{aligned}
{[k] } & =\int_{0}^{L} \rho\left[\phi^{\prime \prime}(x)\right]^{T}\left[\phi^{\prime \prime}(x)\right] d x \\
{\left[k_{s 1}\right] } & =\int_{0}^{L} \rho(L-x)\left[\phi^{\prime}(x)\right]^{T}\left[\phi^{\prime}(x)\right] d x \\
{\left[k_{s 2}\right] } & =\int_{0}^{L} \rho\left(L^{2}-x^{2}\right)\left[\phi^{\prime}(x)\right]^{T}\left[\phi^{\prime}(x)\right] d x \\
{\left[k_{s}\right] } & =R_{d}\left[k_{s 1}\right]+\frac{1}{2}\left[k_{s 2}\right]
\end{aligned}
$$

where $\left(\phi^{\prime}=d \phi / d x\right),[I]$ is the identity modal mass matrix which has developed in this form as result of mode shapes orthogonality condition. $[k]$ is a diagonal matrix that contains the coefficients of the stiffness matrix. The effect of stiffening due to blade rotation appeared in the matrices $\left[k_{s 1}\right],\left[k_{s 2}\right]$ and $\left[k_{s}\right] . R_{d}$ is the disk radius. The vectors $[a],[b]$ represent the inertia coupling between the system torsional flexibility and the blade modal degrees of freedom. 


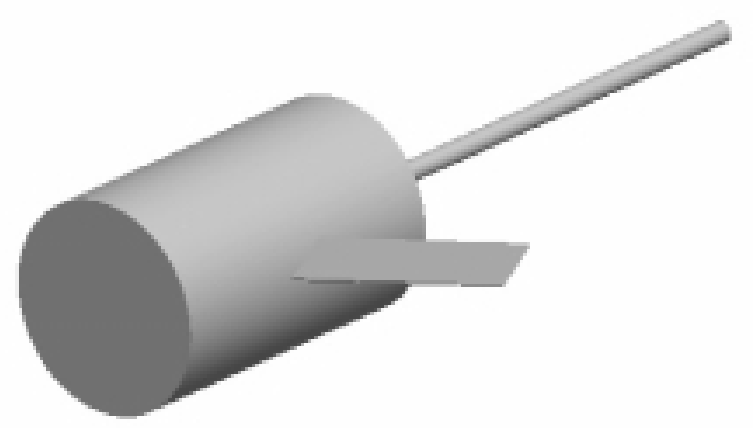

(a) 3D Solid Model
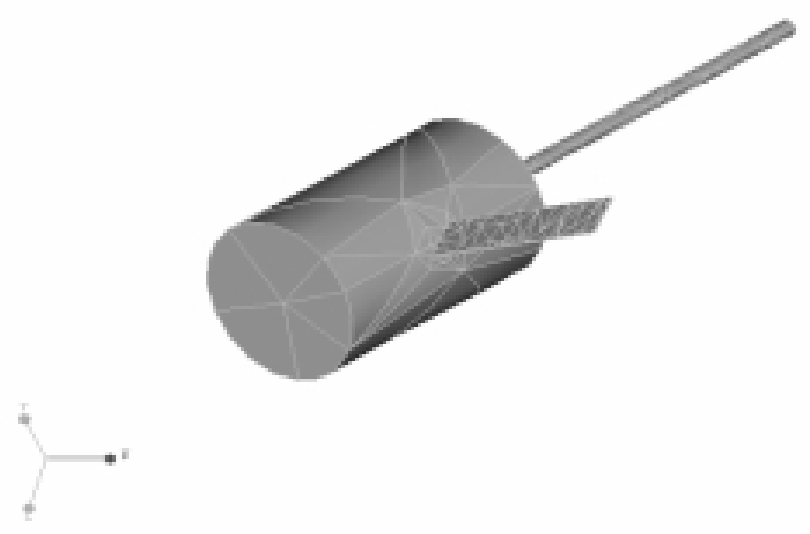

(b) 3D Meshed Model

Fig. 8. 3D Solid and Meshed Models using Pro Mechanica FE Package.

\subsection{The equations of motion}

After substituting the discretized form of the blade deflection given in Eq. (19) and extracting the constant coefficient matrices and vectors, the kinetic and potential energy expressions are used to develop the following expression of the Lagrangian

$$
\begin{aligned}
\tilde{L}= & \frac{1}{2} J_{d}(\omega+\dot{\psi})^{2}+\frac{1}{2} J_{b}\left((\omega+\dot{\psi})^{2}+\omega^{2} \psi^{2}\right)+\frac{1}{2}\left[(\omega+\dot{\psi})^{2}+\omega^{2} \psi^{2}\right] \cos ^{2} \beta\{q\}^{T}[I]\{q\} \\
& +\frac{1}{2}\left(1+\psi^{2} \cos ^{2} \beta\right)\{\dot{q}\}^{T}[I]\{\dot{q}\}+\psi(\dot{\psi}+\omega-\omega \psi) \cos ^{2} \beta\{q\}^{T}[I]\{\dot{q}\}-\frac{1}{2} k_{T} \psi^{2}-\frac{1}{2} \frac{E I}{\rho L^{4}} \\
& \{q\}^{T}[k]\{q\}-\frac{1}{2} \omega^{2}\{q\}^{T}\left[k_{s}\right]\{q\}
\end{aligned}
$$

where $J_{d}=1 / 2 M_{d} R_{d}^{2}$ is the disk moment of inertia, $M_{d}$ and $R_{d}$ are the mass and the radius of the disk, respectively. $J_{b}$ is the blade mass moment of inertia which is found to have the following expression

$$
J_{b}=\rho L\left(R_{d}^{2}+\frac{L^{2}}{3}+R_{d} L\right)
$$

where $\rho$ and $L$ are the blade mass per unit length and blade length, respectively.

Now using the discretized form of the system Lagrangian $\tilde{L}$ in the Lagrange's equation, performing the required differentiation and mathematical manipulation for the system degrees of freedom $(\psi,\{q\})$, neglecting the developed 

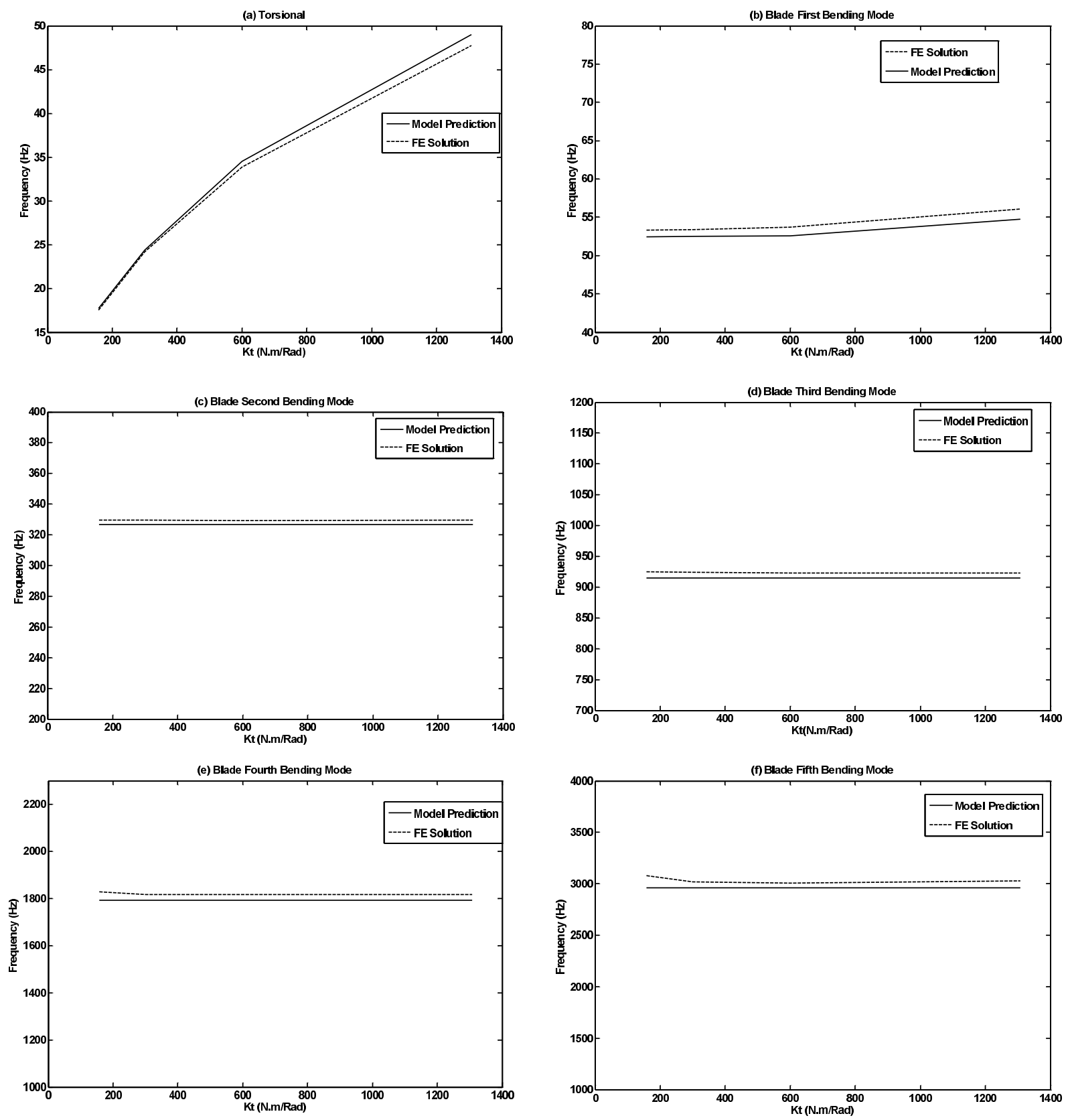

Fig. 9. Natural frequencies as function of $k_{T}$ Comparison for both Predicted Model and FE Solution.

nonlinear damping matrix and dropping terms of quadratic and higher order in the system degrees of freedom (i.e. $\psi^{2},\{q\}^{T}\{q\}$ and $\left.\psi\{q\}\right)$ the linearized system equations of motion can be represented in the following compact matrix form

$$
\begin{array}{cl}
{\left[\begin{array}{cl}
J_{d}+J_{b} & \cos \beta\left[[a]+R_{d}[b]\right] \\
\cos \beta\left[[a]+R_{d}[b]\right]^{T} & {[I]}
\end{array}\right]\left\{\begin{array}{l}
\ddot{\psi} \\
\{\ddot{q}\}
\end{array}\right\}+\left[\begin{array}{cc}
k_{T}-\omega^{2} J_{b} & 0 \\
0 & \frac{E I}{\rho L^{4}}[k]+\omega^{2}\left[\left[k_{s}\right]-[I]\right]
\end{array}\right]} \\
\left\{\begin{array}{l}
\psi \\
\{q\}
\end{array}\right\}=\left\{\begin{array}{l}
0 \\
\{0\}
\end{array}\right\} &
\end{array}
$$



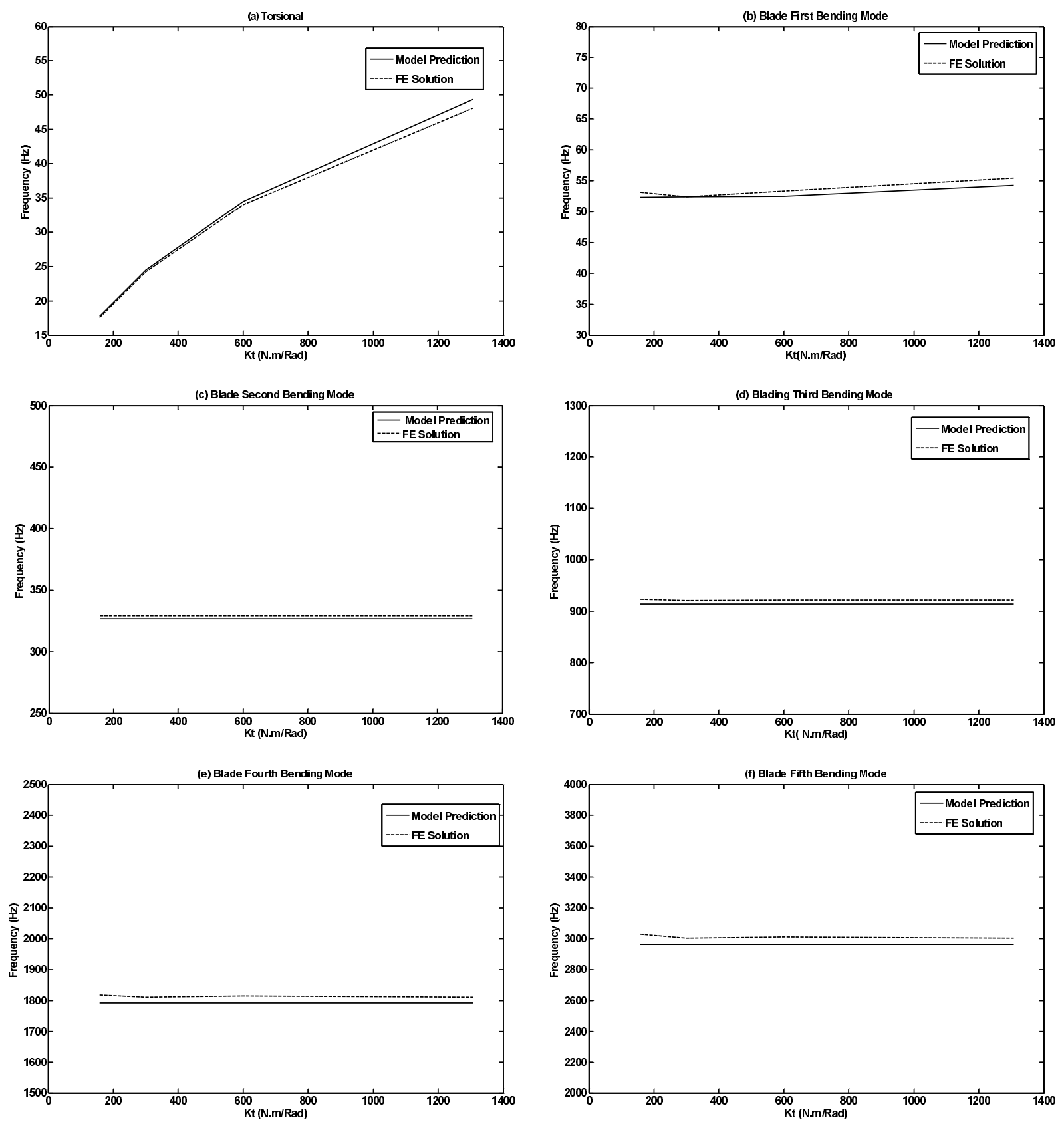

Fig. 10. FEM Model Shaft torsional and blade bending modes for $k_{T}=300 \mathrm{~N} \cdot \mathrm{m} / \mathrm{Rad}, \beta=30^{\circ}$.

Now Eq. (30) represent a standard eignvalue problem for a system that is dynamically coupled. As shown, the coupling term between blade bending modal coordinates $\{q\}$ and the system torsional degree of freedom, $\psi$, is the vector $\cos \beta\left[[a]+R_{d}[b]\right]$ which is dependent on the blade stagger (setting) angle $\beta$, the disk radius $R_{d}$ and the vectors $[a]$ and $[b]$. These coefficient vectors are defined in Eqs (21) and (22), respectively, and shown to be dependent on the mass per unit length and blade length. The other parts of the inertia matrix are composed of the disk inertia, blade inertia and the identity matrix $[I]$ that developed due to the orthogonality of the blade bending modes shape functions. The stiffness matrix shows that the system, as reached in the present formulation, is statically uncoupled. However, the effect of spinning on the system does appear in both stiffness related to the torsional degree of freedom 
Table 1

Blade-disk-shaft data

\begin{tabular}{ll}
\hline Property & Value \\
\hline Blade material & Steel $\left(E=200 \mathrm{GPA}, \hat{\rho}=7850 \mathrm{~kg} / \mathrm{m}^{3}\right)$ \\
Blade length L & $0.125 \mathrm{~m}$ \\
Blade Cross Section & $2.54 \mathrm{X0.1 \textrm {cm }}$ \\
Blade mass per unit length, $\rho$ & $0.2 \mathrm{~kg} / \mathrm{m}$ \\
Blade flexural rigidity, EI & $0.4233 \mathrm{~N} \cdot \mathrm{m}^{2}$ \\
Disk Radius, $R_{D}$ & $0.05 \mathrm{~m}$ \\
Disk moment of inertia, $J_{D}$ & $0.01233125 \mathrm{~kg} . \mathrm{m}^{2}$ \\
Steel & $G=80 \mathrm{GPA}$ \\
shaft Shaft length & $0.5 \mathrm{~m}$ \\
Shaft diameter & $1 \mathrm{~cm}$ \\
Torsional stiffness $k_{T}$ & $157.08 \mathrm{~N} \cdot \mathrm{m} / \mathrm{Rad}$ \\
\hline
\end{tabular}

Table 2

Uncoupled system natural frequencies

\begin{tabular}{ccc}
\hline Degree of Freedom & Natural Frequency $(\mathrm{Hz})$ & FE Solution $(\mathrm{Hz})$ \\
\hline Torsional & 18 & 17.7 \\
Blade $1^{\text {st }}$ bending mode & 52.1 & 52.8 \\
Blade $2^{\text {nd }}$ bending mode & 326.25 & 330.9 \\
Blade $3^{\text {rd }}$ bending mode & 914.271 & 927.81 \\
Blade $4^{\text {th }}$ bending mode & 1791.6 & 1824.28 \\
Blade $5^{\text {th }}$ bending mode & 2961.6 & 3028.29 \\
\hline
\end{tabular}

and the blade bending modal coordinates. As can be seen in the first entry of the stiffness matrix, Eq. (30), spinning works on softening the torsional degree of freedom as related to square of the spinning speed and the mass moment of inertia of the blade in the expression $\left(k_{T}-\omega^{2} J_{b}\right)$. This puts a limit on the combined parameter of spinning speed and the blade mass moment of inertia not to be greater than the torsional stiffness; otherwise the system torsional degree of freedom will be statically unstable. The blade bending modal coordinates are affected both by stiffening and softening as shown. Moreover, the inclusion of the stiffening effect in the form of matrix $\left[k_{s}\right]$ has produced coupling in the stiffness matrix between the individual modes as the matrix $\left[k_{s}\right]$ is non-diagonal.

\section{System natural frequencies}

The natural frequencies for the system are calculated for a system available in the Advanced Mechanics Laboratory (AML) at King Fahd University of Petroleum\& Minerals. The basic dimensions and properties of the blade, disk and shaft are given in Table 1. The results of calculation of the natural frequencies of the uncoupled and non-rotating system are given in Table 2, using the cantilever analytical formula and the Pro-Mechanica finite element solution. The main parameters that affect the system natural frequencies and the coupling are the blade stagger (setting) angle $\beta$, the disk radius $R_{d}$, disk mass $m_{d}$, shaft torsional stiffness $k_{T}$ and the blade mass and length. The effects of these parameters are investigated having mainly the blade parameters as the fixed ones. The basic dimensions and properties given in Table 1 are used except the parameter that is changing to investigate its particular effect in the forthcoming analysis.

The natural frequencies of the shaft-disk-blade system are calculated as function of the shaft torsional stiffness for different setting angles and shown in Fig. 2. The torsional natural frequencies, Fig. 2(a), increases as $k_{T}$ increases with no effect of the blade setting angle. The blade first bending natural frequency, Fig. 2(b), increases as $k_{T}$ increases with higher rate for setting angle $\beta=0^{\circ}$. The rate of increase in the blade first bending frequency decreases as $\beta$ increases until it becomes independent of $k_{T}$ when $\beta=90^{\circ}$. This behavior can be referred to the vanishing of coupling between the blade bending mode and the shaft torsional degree of freedom as they become orthogonal to each other. The blade higher bending natural frequencies are shown in Fig. 2(b-f). Wherein, the effect of torsional stiffness on the blade natural frequencies is shown to be less as we go for higher modes.

The blade-disk-shaft system natural frequencies, for different values of rotating speeds, as function of torsional stiffness $k_{T}$ are calculated and shown in Fig. 3. The shaft torsional natural frequency, Fig. 3(a), shows that there is a 

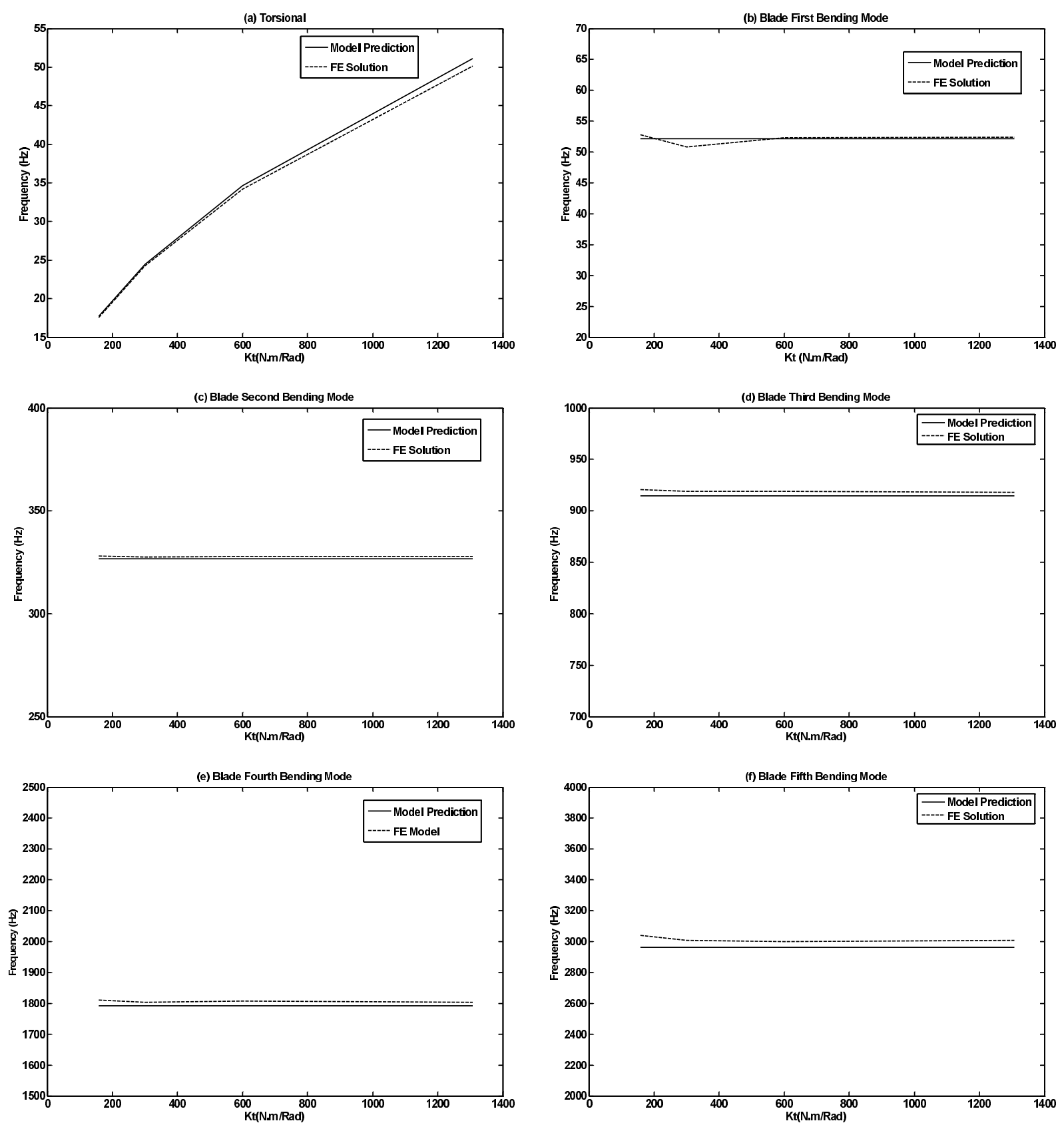

Fig. 11. Natural frequencies as function of $k_{T}$ Comparison for both Predicted Model and FE Solution at $\beta=90^{\circ}$.

minimum value for the torsional stiffness $k_{T}$ below which the system torsional degree of freedom goes unstable due to system rotation softening effect. The variation in blade bending natural frequencies with $k_{T}$ becomes very minor as the rotating speed increases for higher modes. The effect of disk radius $R_{d}$ on the system natural frequencies for different values of setting angle $\beta$ is investigated and the results are given in Fig. 4. As shown the effect of increasing $R_{d}$ is reducing the system natural frequencies for all values of setting angles for the non-rotating system $\omega=0$. The effect of disk radius for the rotating system shown in Fig. 5 is increasing the natural frequencies of the blade bending modes and decreasing the torsional natural frequency. This behavior can be easily referred to the stiffening effect in the blade bending modes and the softening effect in the shaft torsional mode as $R_{d}$ increases. 


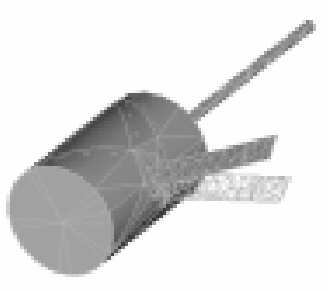

(a) Torsional Mode $(24.2 \mathrm{~Hz})$

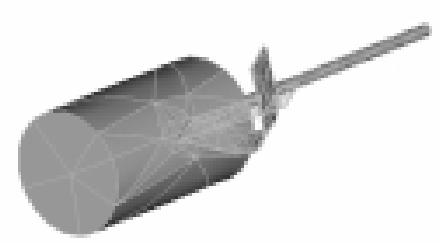

(c) Blade Second Bending Mode $(328.88 \mathrm{~Hz})$
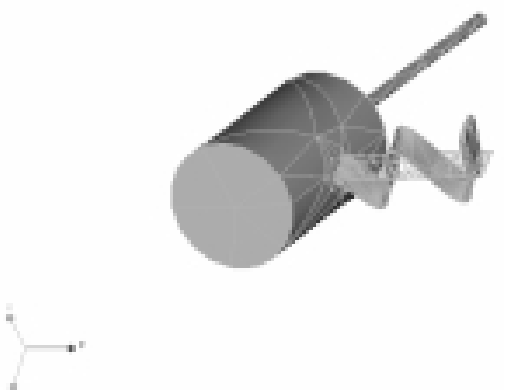

(e) Blade Fourth Bending Mode $(1810.7 \mathrm{~Hz})$
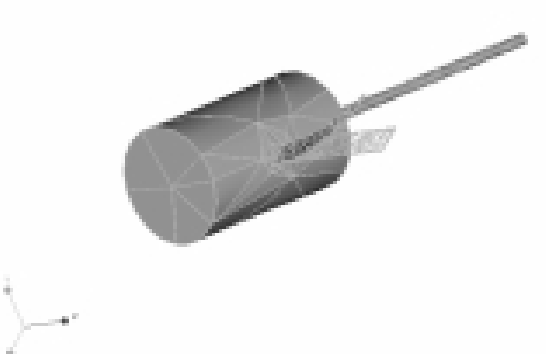

(b) Blade First Bending Mode (52.4 Hz)

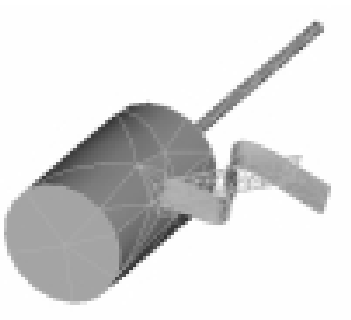

(d) Blade Third Bending Mode $(921.49 \mathrm{~Hz})$

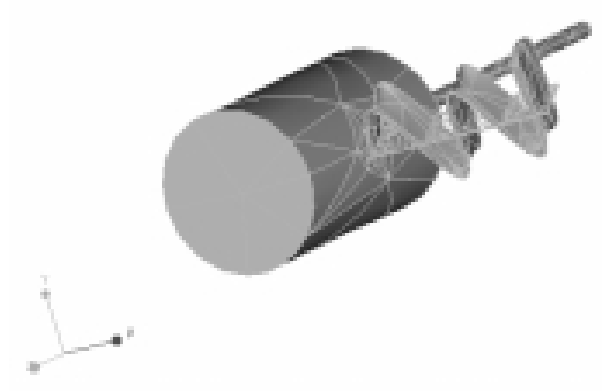

(f) Blade Fifth Bending Mode (3005.32 Hz)

Fig. 12. FEM Model Shaft torsional and blade bending modes for $k_{T}=300 \mathrm{~N} \cdot \mathrm{m} / \mathrm{Rad}, \beta=30^{\circ}$.

The effect of disk mass $M_{d}$ on the system natural frequencies is presented in Fig. 6. The effect is shown to be pronounced at small values of $M_{d}$ and diminishes as $M_{d}$ becomes high relative to the blade inertia. In addition, the effect of setting angle $\beta$ is shown only at small values of disk mass $M_{d}$. The effect of disk mass $M_{d}$ on the system natural frequencies is shown in Fig. 7. Wherein, the increase in disk mass $M_{d}$ is shown to reduce the torsional natural frequencies as expected, Fig. 7(a). The blade first bending natural frequency variation with $M_{d}$ for different values of rotating speeds, Fig. 7(b), shows pronounced effect at low values of $M_{d}$ on the contrary to higher modes that show no effect of $M_{d}$ on the natural frequencies.

To validate the results of the developed reduced order model, the Finite Element Package Pro-Mechanica is used. The solid model and the descritized one are shown in Fig. 8(a) and 8(b), respectively, in the actual dimensions and material properties. The system natural frequencies predicted by the developed model are compared to the Finite Element solution as function of the shaft torsional stiffness and shown in Fig. 9, for setting angle $\beta=0^{\circ}$. Similar comparisons between the model predicted and the Finite Element solution natural frequencies, for setting angles $\beta=30^{\circ}$ and $\beta=90^{\circ}$, in Figs 10 and 11, respectively. Wherein, agreement between the model-predicted and finite element solution natural frequencies is clear. Snapshots of the mode shapes produced by the Finite element solution are given in Fig. 12 for $k_{T}=300$ N.m/Rad and. $\beta=30^{\circ}$. 


\section{Conclusions}

A mathematical model for the shaft-torsional and blade-bending vibration system, rotating at constant speed, is developed in this paper. The blade attached to the rigid disk at a stagger angle from the axis of rotation is modeled using the Euler-Bernoulli beam theory, which in turn is coupled to the system rotation and torsional motions from geometric considerations and using the Lagrangian approach. The obtained equations of motion identified parameters that control the coupling between the shaft torsional and blade vibrations as the stagger angle $\beta$, the disk inertia and the blade inertia. The model is linearized for eignvalue calculation and the natural frequencies are found for different combination of system parameters. Increasing the system speed of rotation and the rotating blade inertia are found to decrease the system torsional natural frequency and play as a softening agent. Moreover, the stagger angle was found to affect the blade natural frequencies rather than the torsional system natural frequency. Small values of disk-radius and disk-mass are shown to increase the blade bending natural frequencies, which one wouldn't expect. The model predicted natural frequencies were validated using a Finite Element Package solution and excellent agreement was found. More studies on modeling other blade vibration modes such as the blade torsional mode are recommended.

\section{Acknowledgments}

The author acknowledges the support of King Fahd University of Petroleum (KFUPM), Dhahran-Saudi Arabia. This work is funded by KFUPM Research Office under project number ME/BLADE-VIBRATION/215. Also the author acknowledges the support of the university of Jordan, Amman-Jordan.

\section{References}

[1] D. Pruelli, Natural bending frequency comparable to rotational frequency in rotating cantilever beam, Journal of Applied Mechanics 39 (1972), 602-604

[2] P.W. Likins, Mathematical modeling of spinning elastic bodies, AIAA Journal 11(9) (1973), 1251-1258.

[3] K. Kaza and R. Kvaternik, Nonlinear flap-lag-axial equations of a rotating beam, AIAA Journal 15(6) (1977), 871-874.

[4] E.F. Crawley and D.R. Mokadam, Stager angle dependence of the inertial and the elastic coupling in bladed disks, Journal of Vibration and Acoustics 106 (1984), 181-188.

[5] E.F. Crawley, E.H. Duchrame and D.R. Mokadam, Analytical and experimental investigation of the coupled bladed disk/shaft whirl of a cantilever turbofan, Journal of Engineering for Gas Turbines and Power 108 (1986), 567-576.

[6] A. Okabe, Y. Otawara, R. Kaneko, O. Matsushita and K. Namura, An equivalent reduced modeling method and its application to shaft-blade coupled torsional vibration analysis of a turbine-generator set, Proc. Of Inst. Mech. Engineers 205 (1991), $173-181$.

[7] S. Huang and K. Ho, Coupled shaft-torsion and blade-bending vibrations of a rotating shaft-blade unit, Journal of Engineering for Gas Turbines and Power 118 (1996), 100-106.

[8] B.O. Al-Bedoor, Dynamic model of coupled shaft torsional and blade bending deformations in rotors, Computer Methods in Applied Mechanics and Engineering 169 (1999), 177-190.

[9] B.O. Al-Bedoor, Reduced-order nonlinear dynamic model of coupled shaft-torsional and blade-bending vibrations in rotors, Journal of Engineering for Gas Turbines and Power 123(1) (2001), 82-88.

[10] B.O. Al-Bedoor, Y. Al-Nassar, L. Ghoutti, S. Aidwesi and M. ABdulSamad, Shaft lateral and torsional vibration responses to blades random vibration excitation, The Arabian Journal for Science and Engineering 29(1C) (2004), 39-67. 

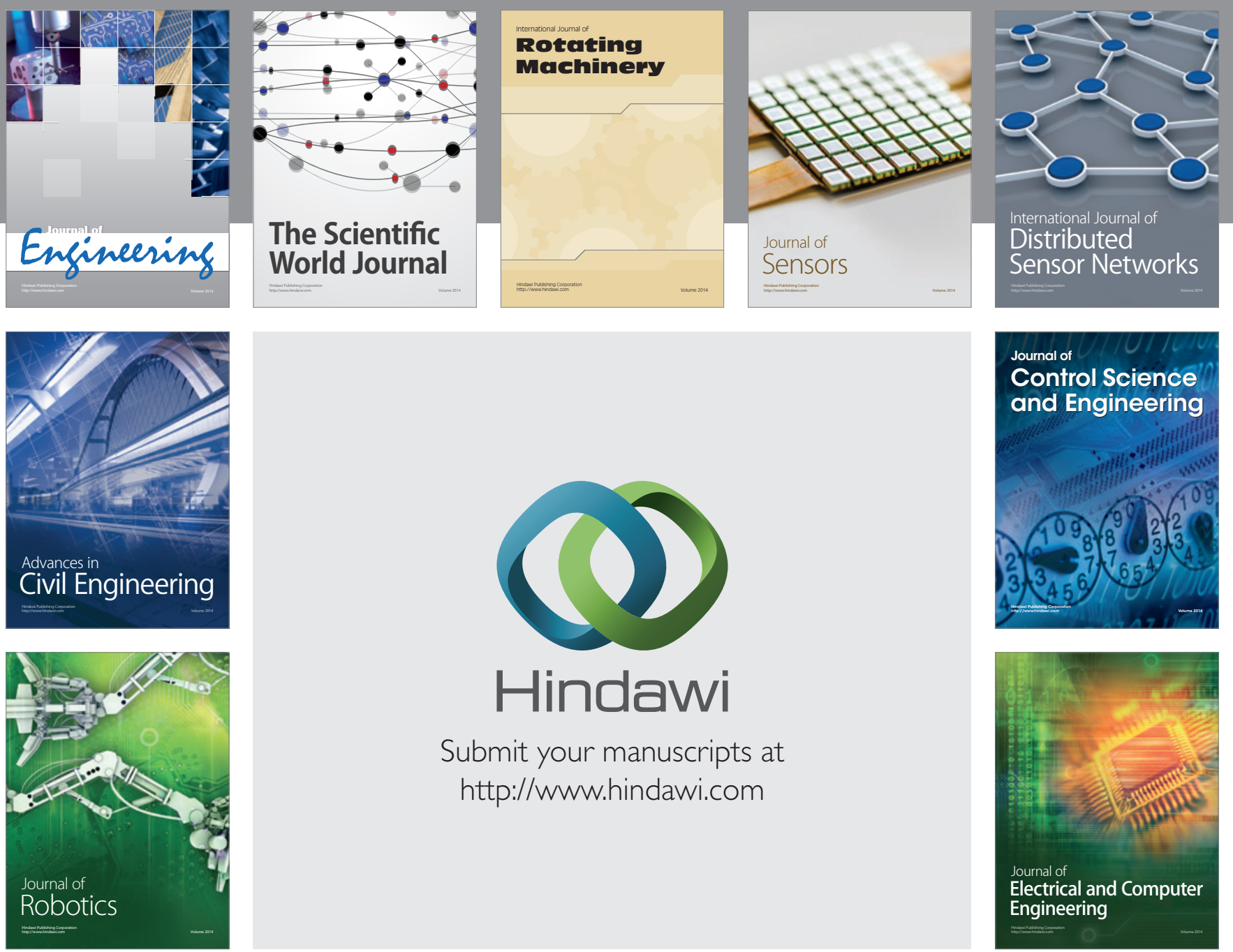

Submit your manuscripts at

http://www.hindawi.com
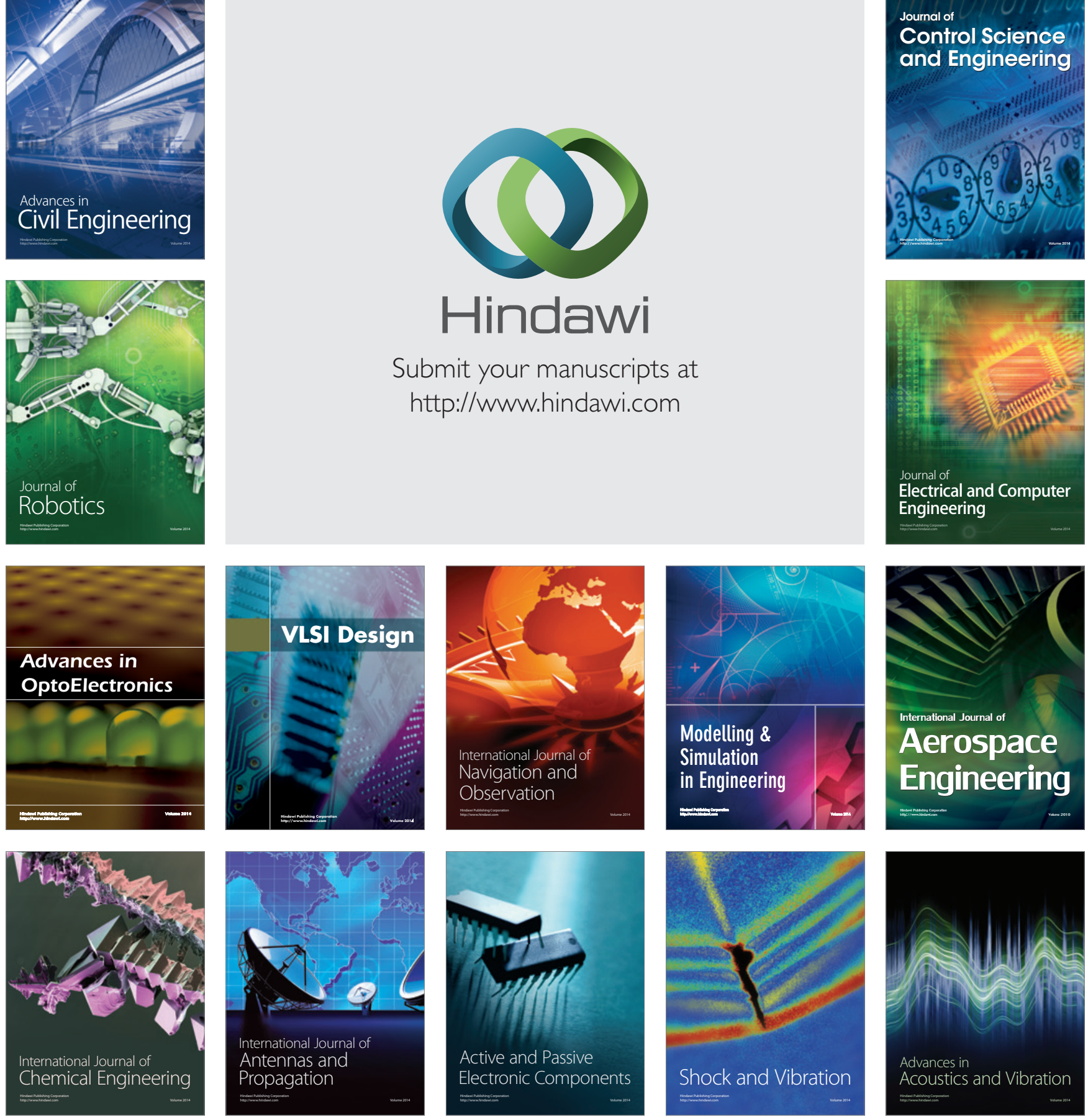